\title{
An algorithm evaluation for discovering classification rules with gene expression programming
}

\author{
Alain Guerrero-Enamorado ${ }^{1}$, Carlos Morell $^{2}$, Amin Y. Noaman ${ }^{3}$, Sebastián Ventura $^{4}$ \\ ${ }^{1}$ Universidad de las Ciencias Informáticas (UCI), \\ Habana, Cuba, \\ E-mail: alaing@uci.cu \\ ${ }^{2}$ Universidad Central de Las Villas (UCLV), \\ Villa Clara, Cuba, \\ E-mail: cmorellp@uclv.edu.cu \\ ${ }^{3}$ King Abdulaziz University, \\ Jeddah, Saudi Arabia \\ E-mail: anoaman@kau.edu.sa \\ ${ }^{4}$ University of Córdoba (UCO), \\ Córdoba, Spain \\ King Abdulaziz University, \\ Jeddah, Saudi Arabia \\ E-mail: sventura@uco.es
}

Received 17 April 2015

Accepted 27 December 2015

\begin{abstract}
In recent years, evolutionary algorithms have been used for classification tasks. However, only a limited number of comparisons exist between classification genetic rule-based systems and gene expression programming rule-based systems. In this paper, a new algorithm for classification using gene expression programming is proposed to accomplish this task, which was compared with several classical state-ofthe-art rule-based classifiers. The proposed classifier uses a Michigan approach; the evolutionary process with elitism is guided by a token competition that improves the exploration of fitness surface. Individuals that cover instances, covered previously by others individuals, are penalized. The fitness function is constructed by the multiplying three factors: sensibility, specificity and simplicity. The classifier was constructed as a decision list, sorted by the positive predictive value. The most numerous class was used as the default class. Until now, only numerical attributes are allowed and a mono objective algorithm that combines the three fitness factors is implemented. Experiments with twenty benchmark data sets have shown that our approach is significantly better in validation accuracy than some genetic rule-based state-of-the-art algorithms (i.e., SLAVE, HIDER, Tan, Falco, Bojarczuk and CORE) and not significantly worse than other better algorithms (i.e., GASSIST, LOGIT-BOOST and UCS).
\end{abstract}

Keywords: Genetic programming, Gene expression programming, Classification rules, Discriminant functions 


\section{Introduction}

From the outset, data mining has acquired great interest. Some lines of research focus on developing algorithms for knowledge extraction from huge databases. Within this rich field, the design of an algorithm for discovering classification rules is one of such lines and is the subject of this work. For several years, many noteworthy approaches have been used to confront this task: decision trees ${ }^{1}$, support vector machines $^{2}$ and probabilistic classifiers ${ }^{3}$ are some of them.

The development of evolutive algorithms for classification tasks have recently become of great interest, with two fundamental paradigms: genetic algorithms $(\mathrm{GAs})^{4}$ and genetic programming $(\mathrm{GP})^{5}$, as alternatives to search efficiently over complex search spaces.

Some examples of how they are applicable in classification tasks can be verified in $\mathrm{in}^{4,6,7,8,9}$. These evolutive algorithms classically follow the principles of "natural selection" and "survival of the fittest" over several generations where operators of mutation and crossover are applied in the population of individuals. Usually, the major disadvantage of genetic classifiers is the intensive consumption of computational resources. Nevertheless, in many real-life cases, where off-line learning is possible, computational consumption may be not a problem.

Gene expression programming ${ }^{10}$ is a technique that combines the advantages of genetic algorithms and genetic programming, while it avoids some of the disadvantages of both. In gene expression programming (GEP) the chromosomes are fixed length linear symbolic strings (genotype) that code computer programs in the form of expression trees of different shapes and sizes (phenotype). In that way, the evolution process is more efficient over this kind of genotype code, while the expressive power of trees is maintained in the phenotype.

In addition, from the perspective of the evolutionary algorithms, there exist two major ways to define individuals and how they conform the final solution; the first, one rule per individual (Michigan $^{7,11}$ ) and the second, one rule-set per individual (Pittsburg ${ }^{12,13}$ ). In the former, an individual encodes a single rule for which the solution is built with one or several individuals. In the second case, an individual is a complete rule set of the problem for which the solution is the best individual. There are a number of mixed perspectives that combine the above approaches; some examples are Iterative Rule Learning ${ }^{14}$ and Genetic CooperativeCompetitive Learning ${ }^{15,16}$. In recent years, evolutionary algorithms (EAs) have been used in classification tasks. The extensive and comprehensive scientific work on state-of-the-art and comparative studies on genetic rule-based systems (GRBS) by Fernandez et al. ${ }^{17}$ and Orriols et al. ${ }^{18}$ is a clear evidence of how useful these kinds of algorithms are. However, there are only few comparisons between GRBS and gene expression programming rule-based systems (GEPRBS) that support the competitiveness of the latter. Therefore, this paper proposes:

- A new algorithm (details in Section 4) for discovering classification rules with GEP Michigan approach. The classifier is constructed as a decision list, sorted by the positive predictive value (PPV). The most numerous class is used as the default class. To avoid over-fitting, a threshold method was employed.

- Evaluation versus nine GRBS algorithms referred by the specialized literature to assess the competitiveness of the algorithm. The way in which our approach significantly improved accuracy compared to other very useful algorithms is shown. Furthermore, an approach that provides an opportunity to improve the reached results in terms of the number of rules in the learned model is presented. In this manner, the competitiveness of the GEP approach for discovering classification rules is empirically demonstrated.

- An algorithm with positive elements of the Tan et al. ${ }^{19}$ and Zhou et al. ${ }^{20}$ algorithms as starting points, avoiding some features which complicate implementation (In the following they will be identified by the first author name). A coveringstrategy (token competition) similar to that used by Tan was employed. It is a powerful idea inspired in the artificial immune system where a memory vector is utilized to produce multiple rules as well as to remove redundant ones. Moreover, the fitness function proposed by Tan is im- 
proved by adding a third factor, thus prioritizing simpleness in the rules discovery process. The GEP encoding used by Zhou's individuals was implemented, but following exactly the Ferreira ${ }^{10,21}$ recommendations and definitions. The proposed algorithm function set uses a reduced and the smallest subset of the Zhou's function set. A fixed arity of 2 was established in order to simplify the implementation of discriminant functions that encodes the individuals in the expression tree form.

The rest of this paper is organized as follows: In Section 2, GEP technology is explained in detail. In Section 3, discriminant functions for classifying a subset of input attributes are described. Besides, we explain how a one-vs-all approach was used to decompose the multi-class problem in several binary classification problems. Section 4 presents the proposed multi-class GEP algorithm. Several features such as function set, terminal set, fitness calculation, token competition and algorithm pseudocode are described in that section. The experimental results achieved by nine GRBS and MCGEP on twenty benchmark real-world data sets are detailed in Section 5. Finally, Section 6 offers conclusions about the strength and weakness of the proposed algorithm and some directions for future work.

\section{Gene Expression Programming}

In this paper, GEP ${ }^{10,21}$ is used as a way of representing individuals, based on GAs and GP. The fundamental difference between these paradigms lies in the nature of individuals: in GA, symbolic fixedlength strings (chromosomes) are used; in GP, individuals are entities of varying size and shape (trees) and in GEP, individuals are also trees but are encoded in a very simple way as symbolic strings of fixed length. Thus individuals have a simple genotypic representation in form of strings and the phenotype is an expression tree (ET) formed by functions in its non-terminal nodes and input values or constants in its terminal nodes. Furthermore, in GEP a way is proposed of transforming the string gene representation in trees such that any valid string generates a syntactically correct tree. In this paper, the phenotype also represents a discriminant function that is used to build a piece of the classifier.

In GEP, genotype may comprise several genes, each one divided into two parts: head and tail. The head of the gene will have a priori a chosen size for each problem and may contain terminal and nonterminal elements. The tail size, which may only contain non-terminal elements, will be determined by the equation $t=h *(n-1)+1$, where $t$ is the tail size, $h$ is the head size and $n$ is the maximum arity (number of arguments) in the non-terminal set. This expression ensures that in the worst case, there will be sufficient terminals to complete the ET. GEP ensures that any change at genotypic level generates a valid tree at the phenotypic level. Valid tree generation is a problem that may arise and should be treated in GP. Moreover, performing the evolutionary process at the genotype level in a fixed-size string such as in genetic algorithms is more efficient than doing so on a tree like in GP; these two are some of the fundamental advantages present in $\mathrm{GEP}^{21}$.

\subsection{Initial population}

The generation of the initial population in GEP is a simple process. It is only necessary to ensure that the head is generated with terminal and non-terminal elements and the tail only with terminal elements, in all cases chosen randomly from the element set (union of terminal and function set).

\subsection{Genetic operators}

In GEP, there are several genetic operators available to guide the evolutionary process, which can be categorized into: mutation, crossover and transposition operators. Sometimes particular GEP transposition operators are included within the mutation category, and other specific GEP operators listed below are included in the crossover category.

\subsubsection{Mutation operator}

As a modifying operator of great intrinsic power, the mutation operator is the most efficient ${ }^{21}$. In GEP, mutations are allowed to occur anywhere in 
the chromosome. However, the structural organization of chromosomes must be preserved. Therefore:

- in the head, any symbol can change into another (from function or terminal set),

- in the tail, terminals can only change into terminals (from terminal set).

\subsubsection{Transposition of insertion sequence (IS) elements}

It starts by randomly selecting a sequence of elements to transpose. This sequence is inserted at a random position at the head of the gene that is being modified, except the beginning of the head as insertion point to avoid generating individuals with only one terminal ${ }^{21}$.

\subsubsection{Root transposition of insertion sequence (RIS) elements}

It starts by selecting a sequence of elements to transpose randomly but with the constraint that starts with a function. A point on the head is randomly chosen for a preselected gene and from this point onwards, the gene is scanned until a function is found. This function becomes the first position of the RIS element. If no function is found, the operator makes no changes. In this way, the RIS operator randomly chooses a chromosome, a gene to be modified and the beginning and end of the RIS sequence. After this, the sequence is inserted into the head of the selected gene ${ }^{21}$.

\subsubsection{One-point recombination}

Two parent chromosomes are placed at the same level and cut from the same point (see Figure 1); then the pieces resulting from each parent are exchanged to generate offspring. The cutoff point is chosen anywhere in the chromosome ${ }^{21}$.

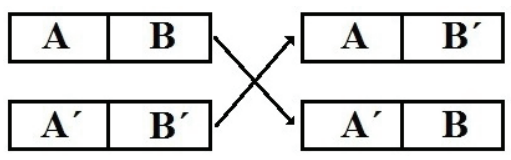

Figure 1: One-point recombination.

\subsubsection{Two-point recombination}

Similar to above but instead of a single cut-off point, two cut-off points are selected from each parent chromosome, which defines a sequence that is exchanged between parents to produce offspring (see Figure 2). With this operator, new blocks are generated, so it is more disruptive than the previous operator. It is recommended in $^{21}$ not to use recombination operators for one and two points alone because they can generate premature convergence. However by combining them with mutation and transposition operators, thorough exploration of the feature space is achieved.

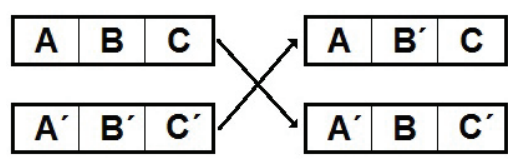

Figure 2: Two-point recombination.

\section{Classification with discriminant functions}

Discriminant functions are one of the schemes used in data mining for classification ${ }^{9}$. In a discriminant function, a subset of the attributes of a pattern is taken for classifying; the output is computed as a value that is the result of evaluating the function at these input attributes. Then, this value must be compared with a threshold (normally 0) to associate it with the corresponding initial pattern classification ${ }^{22}$ (see equation 1) where $\mathrm{X}$ is the input feature vector.

$$
\text { if }(f(X)>0) \text { then } X \in \text { Class }
$$

This classifier will consist of a list of discriminant functions where each function associates an output class. In the case of two classes, the classifier would be as in equation 2 .

$$
\text { if } \begin{array}{r}
(f(X)>0) \text { then } X \in \text { Class }_{1} \\
\text { else } X \in \text { Class }_{2}
\end{array}
$$

In this case, the function $f(X)$ split the characteristic space into two areas, as shown in Figure 3. 


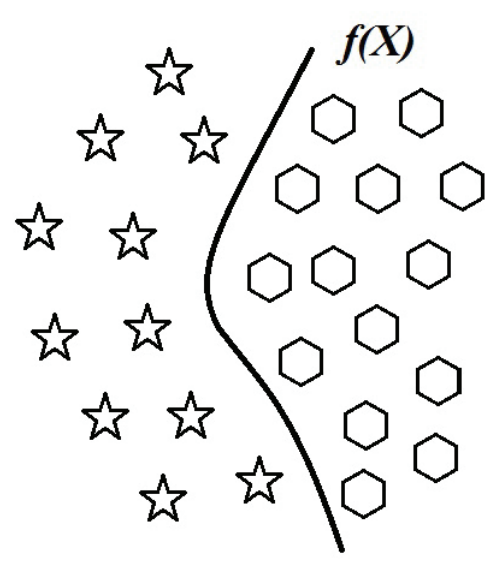

Figure 3: Characteristic space splitting by a discriminant function.

The multi-class problem can be resolved by using a one-vs-all approach ${ }^{23}$, where the $n$-class problem is transformed into $n$ two-class problems. In this approach, the instances of each class are taken as positive instances and the rest as negative instances. On the other hand, a way to build the classifier would be to find a single discriminant function for each class. However, it has the disadvantage that in the real-world problems the features spaces are usually much more complex than Figure 3 , given that one function is generally not able to classify all instances of a determined class properly. Thus, we need to find more than one function for each class: the proposed algorithm searches for discriminant functions to achieve a certain predefined level of coverage over all instances for each class; new functions are added to the solution only when they cover instances not covered by previous functions.

Once the discovery process of discriminant functions concludes, a decision list is generated, with the best functions at the top of the list. When a new instance is presented to this classifier to predict its class, the first function of the list is evaluated. If it returns a positive value, the class associated with this discriminant function is established. If it returns a non-positive value, then the evaluation passes on to the next discriminant function, and so $\mathrm{on}^{24}$. If no discriminant function returns a positive value, the most numerous class in the training set is returned as the default class, as shown in equation 3.

$$
\begin{aligned}
& \text { if }\left(f_{11}(X)>0\right) \text { then } X \in \text { Class }_{1} \\
& \text { elsif }\left(f_{12}(X)>0\right) \text { then } X \in \text { Class }_{1} \\
& \text { elsif }\left(f_{21}(X)>0\right) \text { then } X \in \text { Class }_{2} \\
& \text { elsif }\left(f_{22}(X)>0\right) \text { then } X \in \text { Class }_{2} \\
& \text { elsif }\left(f_{c 1}(X)>0\right) \text { then } X \in \text { Class }_{c} \\
& \text { elsif }\left(f_{c 2}(X)>0\right) \text { then } X \in \text { Class }_{c} \\
& \text { else } X \in \text { Default-Class }
\end{aligned}
$$

Where $f_{i j}$ represents the $j$-th discriminant function that predicts the $i$-th class.

\section{Multi-class with gene expression programming algorithm (MCGEP)}

This classifier is based on a version of the steadystate algorithm with elitism defined in $^{25}$ and it is a modified version of the inspiring algorithm proposed in ${ }^{19}$. In this algorithm, a list of non-redundant individuals is generated with respect to covered instances of the training collection, this process is repeated for each class. To achieve that, a token competition is performed, similar to one proposed by Wong and Leung in ${ }^{26}$.

The fitness of each individual is calculated by multiplying its previous fitness value with the rate between winning tokens and possible-to-win tokens. A token is an instance of the training collection. Covered instances are removed from the competition. Thus, redundant individuals obtain a low fitness score (zero if they cover the same instances covered by others previous individuals in the population). An individual with good initial fitness but with a low rate of new (not covered by others) wontokens may finish the competition with low fitness, even with zero fitness, in the worst case. Before each competition, individuals token-winners are sorted according to their fitness. 


\subsection{Function set and terminal set}

In MCGEP only basic arithmetic functions have been used to build the discriminant functions that form the final classifier as a decision list; the function set was formed by operations: * (multiplication), / (division), + (addition) and - (subtraction); in all cases these functions were established with arity equal to 2 . For the moment, the algorithm has only been developed for numerical data sets; the intention is to make an implementation that works on numerical and non-numerical data sets for greater generality. So the terminals are the attributes of a data set or the constants that were initially defined in the algorithm configuration file. Each individual encodes one rule, representing a discriminant function and having a class as a consequent. The consequent represents the current class in each run of the algorithm, so the algorithm runs as many times as the number of existing classes.

\subsection{Fitness function}

This function (see equation 4) evaluates the fitness of each individual (rule or discriminant function) to be applied to the collection of training; this metric is similar to that used by Bojarczuk et al. in ${ }^{27}$ for a classification rule discovery system with genetic programming.

$$
\begin{aligned}
\text { fitness }= & \frac{t_{p}}{\left(t_{p}+w_{1} * f_{n}\right)} * \frac{t_{n}}{\left(t_{n}+w_{2} * f_{p}\right)} * \\
& * \frac{\text { maxSize }-0.2 * \text { phenot } \text { Size }-0.8}{(\text { maxSize }-1)}
\end{aligned}
$$

where $t_{p}, t_{n}, f_{n}$ and $f_{p}$ represent true positives, true negatives, false negatives and false positives respectively from the confusion matrix obtained by evaluating an individual in the training set; $w_{1}$ and $w_{2}$ are configuration parameters that controls the importance given to false positives and false negatives respectively; this is achieved by setting the function to different contexts. The first factor in the fitness function is also known as sensitivity, the second as specificity. When multiplied, it has also been suggested in $^{28}$, that both factors be used in classifica- tion independently of the paradigm of the algorithm; these factors also have been widely used to measure performance in the domain of medicine ${ }^{29}$. Both factors were weighted by $w_{1}$ and $w_{2}$ like in ${ }^{19}$. When $w_{1}$ decreases and $w_{2}$ increases, the accuracy of the algorithm increases but generates a tendency to overfitting, caused by the increment of the number of rules needed to cover all instances. Tan et al. in ${ }^{19}$ recommended values between 0.2 and 1.0 for $w_{1}$ and between 1.0 and 20 for $w_{2}$.

The third factor is a measure of the simplicity of an individual. This is included because the proposed algorithm does not necessarily produce simple individuals. Although they are limited by the maximum size defined by the GEP chromosome which is maxSize $=2 *\left(\right.$ head $\left._{\text {size }}\right)+1$. The variable phenotSize in the equation 4 is the length (number of terminal and non-terminal elements) of the expression tree coded in the phenotype of an individual; this factor reaches its maximum unitary value when a phenotype is as simplest as possible (phenotype length equal to one). This factor was designed as a negative slope line and decreases to a minimum of 0.8 when length equals the maximum phenotype represented by the value: maxSize. Thus, the most complex individuals are penalized, although not forced to disappear, during the evolutionary process and fitness surface exploration almost obeys the parsimony principle: "the simplest is the best". Thus, fitness function is defined (see equation 4) by multiplying the three factors and then taking values between 0 and 1 .

\subsection{General characteristics of MCGEP algorithm}

To implement the MCGEP algorithm, we reviewed two excellent algorithms for rule discovering, namely Tan et al. ${ }^{19}$ and Zhou et al. ${ }^{20}$. In the Tan algorithm, individuals were encoded like in genetic programming. In Zhou, a GEP variant with a validity test was used. Finally, in MCGEP algorithm, we coded individuals exactly the same way as Ferreira ${ }^{10,21}$ defined them in his work, since it is a much simpler scheme. In the Tan algorithm, the well-known method ramped-half-and-half was used 
Table 1: Comparative table.

\begin{tabular}{|l|l|l|l|}
\hline Features & Tan $2002^{19}$ & Zhou $2003^{20}$ & MCGEP \\
\hline Individual coding & GP & GEP (with validity test) & GEP \\
\hline Initialization method & Ramped-half-and-half & Completely random & $\begin{array}{l}\text { Random, but conserving } \\
\text { head and tail structure }\end{array}$ \\
\hline Function set & AND, NOT & $*,+,-, /$, sqrt, if-then & $*,+,-, /$ \\
\hline Terminal set & $\begin{array}{l}\text { All combinations of } \\
\text { attribute-value (nominal) }\end{array}$ & $\begin{array}{l}\text { Numeric and nominal bi- } \\
\text { narization }\end{array}$ & Numeric \\
\hline Constants set & - & Constant list & Constant list \\
\hline Kind of rules & Classifications rules & Discriminant Functions & Discriminant Functions \\
\hline Kind of classifier & Decision list & Decision list & Decision list \\
\hline $\begin{array}{l}\text { Method to avoid overfit- } \\
\text { ting }\end{array}$ & Support threshold & MDL & Support threshold \\
\hline Fitness function & $F=$ Sen $*$ Esp & $\begin{array}{l}F \quad=\text { Consist Gain } * \\
\text { Complet }\end{array}$ & F=Sen $*$ Esp $*$ Sim \\
\hline Diversity control & Token Competition & - & Token Competition \\
\hline
\end{tabular}

to generate the initial population. In Zhou, the initial population was generated completely randomly.

In MCGEP, we chose the criterion of random generation but kept the head and tail structure, thus avoiding the introduction of new parameters (and adjusting to Ferreira's definitions). In Tan, only logical operations AND and NOT (normal disjunctive form) were used as function set. In Zhou arithmetic $(*,+,-, /$, sqrt) and logical (if $x>0$ then $y$ else $z$ ) operations were used as function set. Again, we used simplicity as the criterion for the MCGEP function set and only used four basic operators $(*,+,-, /)$, all of them with arity $=2$. For Tan, only nominal attributes were allowed, since all combinations of attribute-value were used as the terminal set. Zhou worked with numeric attributes (nominal binarization). For MCGEP, we used the same Zhou scheme (numerical or nominal binarization when needed).

In all cases, the classifier was constructed as a decision list. The Tan method used a threshold to avoid over-fitting. However in Zhou, the MDL (Minimal Description Length) principle was used, with a value $W=0.5$. In our case, although we found Zhou's approach with MDL very interesting, we used the threshold method, which is far simpler and provides very good results (scant over-fitting). On the other hand, as Bacardit ${ }^{30}$ poses in chapter 8 of his report, the $W$ parameter setting becomes very sensitive product selection pressure of genetic algorithms. Making for high values a collapse of the final classifier into one with a single rule; for small values of the parameter, the classifier fails to eliminate over-fitting. Later, Bacardit states that it is no simple task to automatically adjust this parameter, so its inclusion in our algorithm is left for future implementations.

The Tan algorithm used a fitness function with the product of sensitivity and specificity. In Zhou, the product of rule consistency by completeness gain ratio (covered entities) was used. In our case, we modulated the products of sensitivity and specificity with a third factor (which prioritizes the simplicity of the rules). To control diversity, Tan used a token competition. In Zhou, the instances covered are progressively removed from the data set. In our case, we used the method that Tan employs in his algorithm to adjust the fitness of individuals, depending of the new instances covered. In this way, the algorithm tends to eliminate redundancy, while the more general rules are prioritized.

Table 1 shows a comparison of the basic characteristics of MCGEP and the two fundamental works that inspired its development: Tan and Zhou. 


\subsection{Pseudo-code algorithm description}

As shown in the line 4 of the pseudo-code (Algorithm 1), the evolutionary process begins by generating the initial population bset(0). This initial population evolves for several generations until the stop condition is reached -in our algorithm, that means until the maximum number of generations $g_{\max }$ is achieved (this parameter is defined in the algorithm configuration file) or an individual with fitness $\approx 1$ obtained. In each generation, individuals are evaluated according to the performance function defined in 4.2 (see equation 4). Then a small elite percentage population is chosen, which is defined in the configuration file by the parameter elitist $_{\text {perc }}$ and saved in (cset) (see line 8 of pseudo-code). A selection of parents is performed on the remaining population, with two of tournament size, tourn $_{\text {size }}=2$ (see line 10); to select the parents (pset), the GEP genetic operators detailed in section 2.2 were applied one by one (see lines 11 to 15); thereafter, the offspring obtained (in rset) were evaluated (see line 16). Subsequently, a reproduction process where some individuals from the original bset population are randomly chosen with a low probability defined in the configuration file as copy $_{\text {prob }}=0.1$ and bound to the previously obtained elite population (cset) (see line 17); finally individuals: cset, eset and rset are joined in a unique population cset (initially eset is empty), as can be seen in lines 18 and 19. Then, a TokenCompetition on cset population is executed (see line 20), the competition returns a vector of rules that are then stored in eset which is a potential classifier for the current class, the eset rules were obtained from individuals who had a reason (CoveredTokens/TotalTokens) $>$ threshold $_{\text {support }}$, then eset population is re-evaluated to prepare for the next generation (see line 21), in the line 22 , the population is adjusted back to the original size defined by pop size. The whole process is repeated generation after generation from line 6 until it reaches the maximum number of generations, or an individual achieves a fitness very close to 1.0. If any of the above stop conditions is reached, all the elements of eset are added to the classifier and the algorithm starts with the next class, repeating the whole process from line 3 onwards.

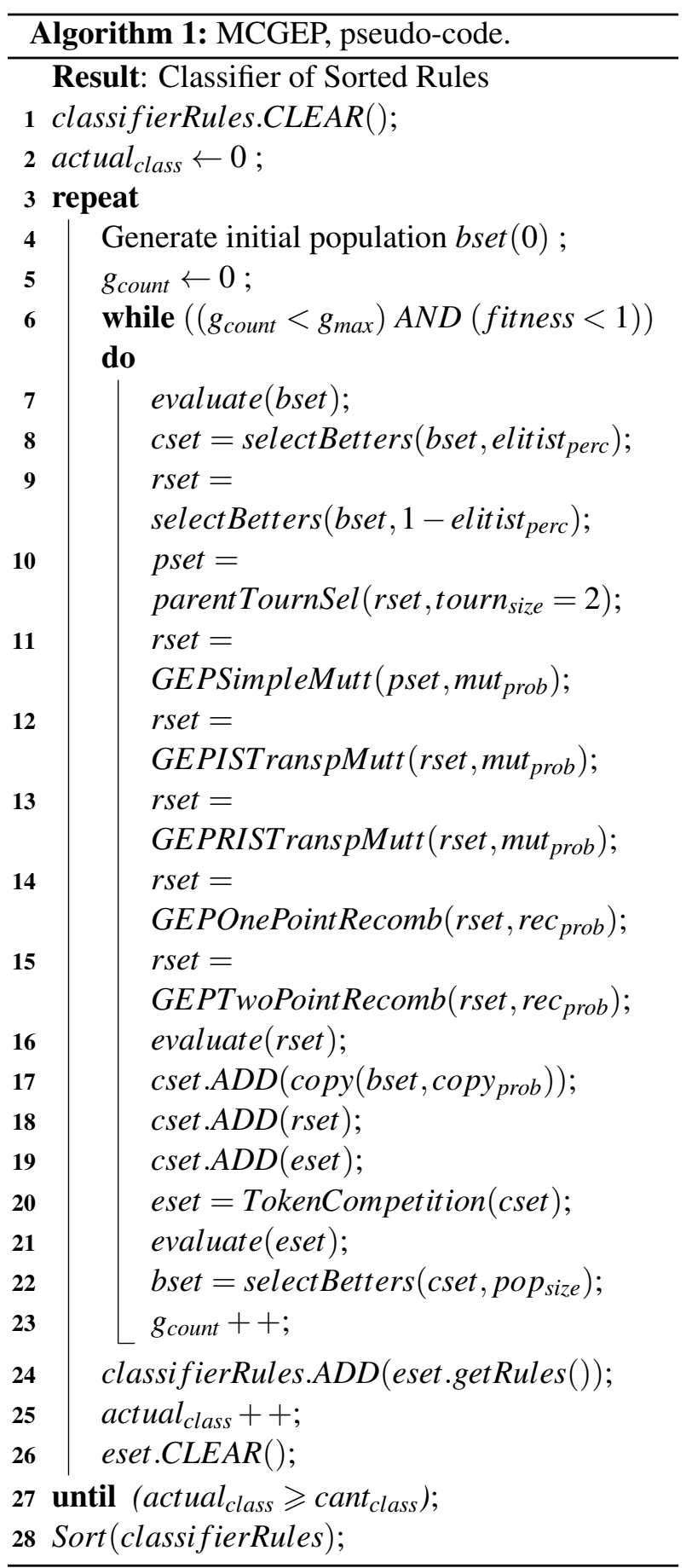

The class used for fitness calculation is assigned in each algorithm iteration as a rule consequent, assuming as positive instances those belonging to that class and as negative those belonging to other classes. The algorithm is repeated as many times as the number of existing classes in the training set. All 
the rules coded in eset individuals are added to classifier in each cycle. The list eset is reset each time; see line 26. At the end of the process, we sort all rules in the classifier according to the PPV value. In each sorting cycle, we add the PPV-best rule to the end of a list of rules and remove all the matching instances from the training set. We repeat this process until the training set is empty or no more rules for sorting (we initially used an empty list of rules and the full training set to calculate the first PPV-best rule). PPV is computed as $P P V=t_{p} /\left(t_{p}+f_{p}\right)$.

\section{Empirical evaluation}

This section presents the experimental methodology followed to evaluate the algorithms presented. The accuracy and the size of the models evolved by all algorithms involved in this work is compared. The competence of the MCGEP is compared with nine widely-known GP techniques for discovering classification rules that represent a large variety of learning paradigms. The methods used in the comparison are:

1. $\mathrm{UCS}^{31}$ : genetic rule-based Michigan-style algorithm derived from $\mathrm{XCS}^{32}$ but specialized for supervised learning tasks. Evolves a best action map more efficiently than XCS complete action maps.

2. GASSIST ${ }^{30}$ : inherits from the GABIL algorithm ${ }^{33}$ and it is one of the most competitive current Pittsburgh-style systems. It is a genetic rule-based system that evolves individuals consisting of an ordered, variablelength rule set.

3. HIDER ${ }^{34}$ : genetic rule-based algorithm that represents knowledge in a similar fashion to GASSIST as a hierarchical set of rules which take the form of a decision list. The main difference of HIDER is that it uses natural coding ${ }^{35}$ to represent each rule. It uses an iterative rule learning approach.

4. SLAVE $^{36}$ : genetic fuzzy-rule-based classifier that implements iterative rule learning with the search space reduction objective.
5. Fuzzy LOGIT-BOOST ${ }^{37}$ : a boosting method similar to AdaBoost ${ }^{38}$ that uses a greedy version of generalized backfitting ${ }^{39}$ to build an additive model. LOGIT-BOOST was extended to induce fuzzy classifiers, resulting in Fuzzy LOGIT-BOOST.

6. $\mathrm{CORE}^{40}$ : in the co-evolutionary algorithm for rules discovery, each individual codifies a rule and the whole rule set is evolved simultaneously. Thus, rules should cooperate with each other to produce an optimal rule set jointly, and at the same time, the rule set is reduced by a token competition.

7. Bojarczuk ${ }^{27}$ : a hybrid Pittsburgh/Michigan approach, where an individual can contain multiple classification rules that predict the same class. An individual is in disjunctive normal form (DNF). An individual consists of a logical disjunction of rule antecedents, where each rule antecedent is a logical conjunction of conditions (attributevalue pairs). This is a genetic programming rule-based system.

8. Falco ${ }^{41}$ : Michigan-style genetic algorithm able to extract classification rules. Each rule is constituted by a logical combination of predicting attributes. It is a grammar guided genetic programming based system.

9. $\operatorname{Tan}^{19}$ : extends the tree representation of genetic programming to evolve multiple comprehensible IF-THEN classification rules in a Michigan-style. It employs a covering algorithm (token competition) inspired by the artificial immune system, where a memory vector is utilized to produce multiple rules, as well as to remove redundant rules. It is a grammar guided genetic programming-based system.

A more detailed explanation of each of the first six algorithms above can be found condensed in an excellent review ${ }^{18}$.

The following subsection, provides details of the real-world problems chosen for the experiments, 
the experimental tools and configurations parameters for each one, the experimental results and the statistical analysis applied to compare the results obtained.

\subsection{Training sets}

To evaluate the behavior of our classifier, twenty real-world data sets were chosen from KEEL ${ }^{* 42}$ and $\mathrm{UCI}^{\dagger 43}$ repositories.

Table 2: Data set characteristics.

\begin{tabular}{|c|c|c|c|c|}
\hline Id. & Data set & Instances & Attrib. & Class \\
\hline aus & Australian & 690 & 14 & 2 \\
bal & Balance & 625 & 4 & 3 \\
bup & Bupa & 345 & 6 & 2 \\
cle & Cleveland & 297 & 13 & 5 \\
con & Contraceptive & 1473 & 9 & 3 \\
der & Dermatology & 358 & 34 & 6 \\
eco & Ecoli & 336 & 7 & 8 \\
gla & Glass & 214 & 9 & 7 \\
hea & Heart-s & 270 & 13 & 2 \\
iri & Iris & 150 & 4 & 3 \\
pim & Pima & 768 & 8 & 2 \\
son & Sonar & 208 & 60 & 2 \\
tex & Texture & 5500 & 40 & 11 \\
thy & Thyroid & 7200 & 21 & 3 \\
wdb & Wdbc & 569 & 30 & 2 \\
win & Wine & 178 & 13 & 3 \\
wis & Wisconsin & 683 & 9 & 2 \\
wpb & Wpbc & 194 & 32 & 2 \\
yea & Yeast & 1484 & 8 & 10 \\
zoo & Zoo & 101 & 17 & 7 \\
\hline
\end{tabular}

UCI is a well-known repository and KEEL is an open source Java software tool which empowers the user to assess the behavior of evolutionary learning and Soft Computing based techniques. In particular, KEEL-dataset includes the data set partitions in the KEEL format classified as: regression, clustering, multi-instance, imbalanced classification, multi-label classification and so on. To ex-

\footnotetext{
* http://www.keel.es/

$\dagger$ http://archive.ics.uci.edu/ml/

$\ddagger$ http://jclec.sourceforge.net/
}

ecute the experiments, the following data sets were used: Australian, Balance, Bupa, Cleveland, Contraceptive, Dermatology, Ecoli, Glass, Heart, Iris, Pima, Sonar, Texture, Thyroid, Wdbc, Wine, Wisconsin, Wpbc, Yeast and Zoo. In our experimental assessment, all examples with missing values were removed from the data sets. In total, we have eight binary problems, five three-class problems and seven problems between 5 and 11 class. A summary of the characteristics of these data sets can be seen in the Table 2.

\subsection{Experimental tools}

We used the JCLEC ${ }^{\ddagger}$ framework described in ${ }^{44}$; it is a software system for Evolutionary Computation (EC) research, developed in Java programming language.

Table 3: MCGEP sumary parameters.

\begin{tabular}{|l|c|}
\hline Parameter & Value \\
\hline Population Size (population-size) & 500 \\
Max of Generations (max-of-generations) & 100 \\
Tournament selector (tournament-size) & 2 \\
Copy probability (copy-prob) & 0.1 \\
Elite probability (elitist-prob) & 0.1 \\
Support (support) & 0.01 \\
Parameters ( $w_{1} ; w_{2}$ ) & $(1 ; 2)$ \\
GEPSimpleMutator (mut-prob) & 0.15 \\
GEPISTranspositionMutator (mut-prob) & 0.10 \\
GEPRISTranspositionMutator (mut-prob) & 0.10 \\
GEPOnePointRecombinator (rec-prob) & 0.40 \\
GEPTwoPointsRecombinator (rec-prob) & 0.40 \\
\hline
\end{tabular}

It provides a high-level software framework to perform any kind of Evolutionary Algorithm (EA), providing support for genetic algorithms (binary, integer and real encoding), genetic programming (Koza's style, strongly typed, and grammar based) and evolutionary programming. It has a module for classification, where the MCGEP algorithm described in Section 4 was implemented, GEP genetic 
operators of JCLEC were used. The evaluator was implemented in JCLEC with the fitness function described in section 4.2. Furthermore, to perform the simulations, a cluster with Multi-Core $12 \times 2.4 \mathrm{GHz}$ processors, 23.58 GB of RAM and Linux 2.6.32 as the operating system were used. The parameters used in the algorithm are summarized in Table 3. They were kept constant during the experiments. In our experiments, we used the chromosome size and constants list dependent on the characteristics of the problem. The list of constants is chosen depending on the range of attribute values (only in glass, heart$\mathrm{s}$, pima, wdbc and wine). The configuration files for MCGEP and complementary material of the experimental study can be consulted at the link

Table 4: MCGEP problem configuration.

\begin{tabular}{|c|c|c|}
\hline Dataset & Head $_{\text {size }}$ & Const. List \\
\hline Australian & 140 & $1 ; 0.5$ \\
Balance & 25 & $1 ; 0.5$ \\
Bupa & 40 & $1 ; 0.5$ \\
Cleveland & 130 & $1 ; 0.5$ \\
Contraceptive & 90 & $1 ; 0.5$ \\
Dermatology & 340 & $1 ; 0.5$ \\
Ecoli & 70 & $1 ; 0.5$ \\
Glass & 100 & $1 ; 0.5 ; 5 ; 50$ \\
Heart-s & 150 & $1 ; 0.5 ; 5 ; 50$ \\
Iris & 40 & $1 ; 0.5$ \\
Pima & 60 & $1 ; 0.5 ; 10 ; 400$ \\
Sonar & 65 & $1 ; 0.5$ \\
Texture & 400 & $1 ; 0.5$ \\
Thyroid & 180 & $1 ; 0.5$ \\
Wdbc & 300 & $1 ; 0.5 ; 50 ; 500$ \\
Wine & 130 & $1 ; 0.5 ; 50 ; 500$ \\
Wisconsin & 90 & $1 ; 0.5$ \\
Wpbc & 80 & $1 ; 0.5$ \\
Yeast & 80 & $1 ; 0.5$ \\
Zoo & 170 & $1 ; 0.5$ \\
\hline
\end{tabular}

How the size of the head in GEP is defined, remains an open issue ${ }^{10,45}$, so we adjusted this parameter through trial and error starting with values close to ten times the number of attributes of each data set. To achieve repeatability of the experiments, we show the configurations of each problem in Tables 3 and 4 . The source codes and optimum configurations for UCS, GASSIST, HIDER, SLAVE, LOGITBOOST and CORE were based in KEEL ${ }^{46}$, its original authors and in the useful review paper ${ }^{18}$. We used the JCLEC implementation of the Bojarczuk, Falco and Tan algorithms, with the configurations recommended by the original authors ${ }^{19,27,41}$ respectively.

\subsection{Experimental results}

We evaluated the performance of the models evolved by each learning system with the test accuracy metric (proportion of correct classifications over previously unseen examples) and number of rules of the models obtained in each case. We used a ten-fold cross validation procedure with 5 different random seeds over each data set. The average results for each collection are shown in Table 5, for accuracy and in Table 6 for number of rules. The last row in Tables 5 and 6 shows the average rank for each algorithm.

\subsection{Statistical analysis}

We followed the recommendations pointed out by Demšar $^{47}$ to perform the statistical analysis of the results. As he suggests, we used non-parametric statistical tests to compare the accuracies and sizes of the models built by the different learning systems. To compare multiple learning methods, we first applied a multi-comparison statistical procedure to test the null hypothesis that all learning algorithms obtain the same results on average. Specifically, we used Friedman's test. The Friedman test rejected the null hypothesis, so post-hoc Bonferroni-Dunn tests were applied.

According to the results obtained (see Tables 5 and 6) we statistically analyzed the results to detect significant differences between the accuracy and the size of the models evolved by the different learning methods.

The multi-comparison Friedman's test rejected the null hypotheses that (i) all the systems performed the same on average with $p=4.37 \times 10^{-11}$,

\footnotetext{
$\S$ http://www.uco.es/grupos/kdis/kdiswiki/MCGEP/
} 
A. Guerrero-Enamorado et al. / An algorithm evaluation for discovering classification rules

Table 5: Algorithm accuracy and rank over data sets.

\begin{tabular}{|c|c|c|c|c|c|c|c|c|c|c|}
\hline Data set & MCGEP & UCS & $\begin{array}{l}\text { GAS- } \\
\text { SIST }\end{array}$ & HIDER & SLAVE & $\begin{array}{l}\text { LOGIT- } \\
\text { BOOST }\end{array}$ & CORE & $\begin{array}{l}\text { Bojarc- } \\
\text { zuk }\end{array}$ & Falco & Tan \\
\hline aus & $85.57(2)$ & $84.12(6)$ & $86.43(1)$ & $1.65(8)$ & $70.29(10)$ & $83.59(7)$ & $78.67(9)$ & $85.51(3.5)$ & $85.51(3.5)$ & 4.58( \\
\hline bal & $76(1)$ & $.65(5)$ & $.88(3)$ & (7) & (4) & (2) & (8) & & & $71.68(($ \\
\hline bup & $34(1)$ & $.12(3)$ & $.86(5)$ & $.33(4)$ & $.21(8)$ & $69.28(2)$ & (9) & 0) & & \\
\hline cle & $.43(3)$ & $53.03(8)$ & $56.19(1)$ & $53.17(6)$ & $3.63(5)$ & $53.88(4)$ & $53.00(9)$ & (10) & 53.0 & $55.34(2)$ \\
\hline con & $.05(4)$ & $48.19(7)$ & $54.58(1)$ & $52.52(3)$ & $3.82(9)$ & $53.38(2)$ & $44.2 ?$ & 49.0 & $42.53(10)$ & $48.47(6)$ \\
\hline der & $4.95(2)$ & $90.57(5)$ & $95.96(1)$ & 88.54(6) & $0.74(4)$ & $31.03(10)$ & 31.7 & & 73.87(8) & $93.32(3)$ \\
\hline & 72.11(7) & 79.30(3) & 77.23(4) & 75.74(5) & & & & & & $72.22(6)$ \\
\hline & & 72.66 & 66.82 & & & & & & & \\
\hline & (1) & $77.78(5)$ & .78 & & & & & & & 76. \\
\hline ir & $53(1)$ & $94.80(7)$ & $96.40(2)$ & 96.00 & 95.3 & $96.00(4.5)$ & 94.5 & 10) & $8.5)$ & $96.13(3)$ \\
\hline pim & $05(3)$ & 71.78(9) & $73.88(4)$ & 74.2 & & & & & & $72.07(8)$ \\
\hline so & $.38(2)$ & $66.84(7)$ & $76.10(1)$ & $50.78(10)$ & $70.98(5)$ & & & 72.0 & & $68.49(6)$ \\
\hline tex & $03(4)$ & $93.77(1)$ & $62.88(6)$ & & & & & & & $67.31(5)$ \\
\hline & & & & & & & & & & \\
\hline & & 94.73 & 93 & & & & & & & \\
\hline & $03(3)$ & $96.16(2)$ & 93.90(4) & 79 & 5) & & & 3) & & $91.44(6$ \\
\hline & $55(1)$ & $96.36(2)$ & $95.59(4)$ & & & (10) & & & & $95.33(6$ \\
\hline wpb & $3.63(3)$ & $71.45(5)$ & $70.61(6)$ & $64.43(8)$ & 72.83(4) & $69.79(7)$ & $76.32(1)$ & $58.07(10)$ & 75.73(2) & $62.12(9)$ \\
\hline yea & $.33(5)$ & $55.38(3)$ & $55.21(4)$ & $55.79(2)$ & $49.06(6)$ & $58.86(1)$ & $35.86(9)$ & & $35.00(10)$ & $36.60(8)$ \\
\hline zoo & $95.62(3)$ & $94.71(5)$ & $92.84(7)$ & $95.67(2)$ & & & & & & $95.00(2$ \\
\hline Rank & 2.60 & 4.65 & 3.30 & 5.33 & 5.10 & 4.53 & 8.18 & 8.13 & 7.35 & 5.85 \\
\hline
\end{tabular}

Table 6: Number of rules in models obtained over data sets.

\begin{tabular}{|l|l|l|l|l|l|l|l|l|l|l|}
\hline Data set & MCGEP & UCS & $\begin{array}{l}\text { GAS- } \\
\text { SIST }\end{array}$ & HIDER & SLAVE & $\begin{array}{l}\text { LOGIT- } \\
\text { BOOST }\end{array}$ & CORE & $\begin{array}{l}\text { Bojarc- } \\
\text { zuk }\end{array}$ & Falco & Tan \\
\hline aus & 10.44 & 6355.30 & 7.36 & 13.96 & 7.72 & 50.00 & 6.68 & 2.00 & 2.00 & 13.62 \\
bal & 8.58 & 5406.76 & 8.84 & 5.00 & 22.64 & 50.00 & 4.44 & 4.44 & 3.00 & 18.94 \\
bup & 13.06 & 6074.14 & 7.60 & 6.52 & 5.94 & 50.00 & 3.96 & 2.04 & 2.00 & 18.60 \\
cle & 19.84 & 6366.20 & 6.88 & 23.58 & 37.40 & 50.00 & 6.56 & 5.56 & 5.00 & 25.48 \\
con & 24.92 & 6397.38 & 7.90 & 13.84 & 46.66 & 50.00 & 6.12 & 3.02 & 3.00 & 32.36 \\
der & 11.60 & 6162.16 & 6.66 & 10.74 & 10.96 & 50.00 & 1.00 & 6.72 & 6.00 & 11.60 \\
eco & 19.32 & 6154.64 & 6.04 & 12.54 & 13.06 & 50.00 & 6.40 & 9.64 & 8.00 & 25.22 \\
gla & 17.22 & 6294.60 & 5.34 & 25.00 & 15.74 & 50.00 & 6.24 & 7.84 & 7.02 & 20.98 \\
hea & 9.60 & 6300.64 & 6.52 & 9.08 & 7.60 & 50.00 & 7.68 & 2.00 & 2.00 & 15.50 \\
iri & 5.18 & 3184.18 & 4.08 & 4.00 & 3.00 & 50.00 & 3.28 & 3.96 & 3.00 & 6.04 \\
pim & 12.62 & 6359.64 & 8.26 & 12.28 & 10.04 & 50.00 & 4.86 & 2.00 & 2.76 & 19.10 \\
son & 13.04 & 6384.58 & 5.24 & 179.00 & 7.98 & 50.00 & 1.00 & 2.00 & 2.00 & 16.04 \\
tex & 25.96 & 6400.00 & 11.12 & 89.74 & 35.14 & 50.00 & 1.00 & 15.86 & 11.02 & 35.62 \\
thy & 7.40 & 6399.74 & 5.70 & 3.16 & 6.76 & 50.00 & 2.14 & 3.46 & 3.00 & 9.66 \\
wdb & 6.74 & 6351.00 & 5.24 & 99.20 & 5.32 & 50.00 & 2.56 & 2.00 & 2.00 & 9.20 \\
win & 7.46 & 6326.84 & 4.24 & 30.82 & 3.96 & 50.00 & 3.76 & 3.00 & 3.00 & 9.20 \\
wis & 4.56 & 5743.26 & 4.18 & 3.10 & 6.08 & 50.00 & 6.40 & 2.00 & 2.00 & 6.88 \\
wpb & 12.34 & 6359.62 & 5.26 & 131.46 & 9.90 & 50.00 & 1.38 & 2.00 & 2.00 & 18.42 \\
yea & 45.62 & 6397.56 & 7.06 & 48.88 & 23.68 & 50.00 & 5.46 & 12.08 & 10.00 & 51.66 \\
zoo & 7.54 & 5822.30 & 7.10 & 7.60 & 7.30 & 50.00 & 7.74 & 9.08 & 7.00 & 8.40 \\
\hline Rank & 6.08 & 10.00 & 3.75 & 6.25 & 5.28 & 8.75 & 2.73 & 2.90 & 1.80 & 7.48 \\
\hline
\end{tabular}


(ii) the number of rules in the models was equivalent on average with $p=7.28 \times 10^{-11}$. Afterwards, we applied the post-hoc Bonferroni-Dunn test using KEEL tools to detect (i) which learning algorithm performed equivalently to the best-ranked method (MCGEP) according to the test accuracy metric; (ii) which methods evolved models whose number of rules were equivalent to the best ranked method (Falco) according to the model size. Using $\alpha=0.10$ in equation 5 with $\mathrm{k}$, number of algorithms, $q_{\alpha}=2.539$ and $\mathrm{N}$, number of data sets, a critical difference of 2.4309 is obtained.

$$
C D=q_{\alpha} \sqrt{\frac{k(k+1)}{6 N}}
$$

Figure 4 shows the results of this BonferroniDunn test, comparing all systems in terms of both accuracy and the number of rules in the model. The major advantage of this test is that it seems to be easier to visualize, because it uses the same critical difference for all comparisons ${ }^{47}$.

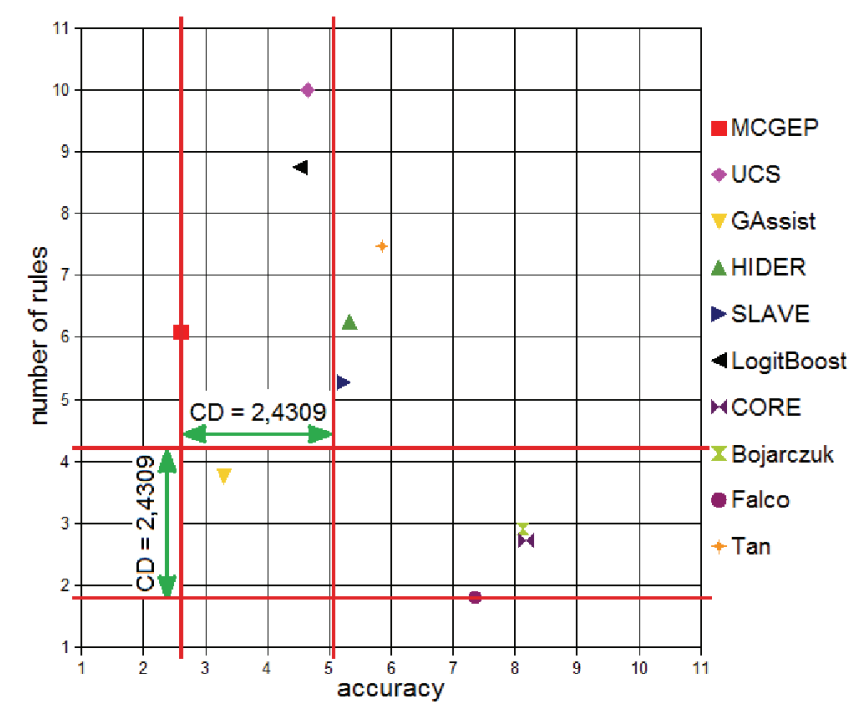

Figure 4: Critical difference comparison of accuracy and number of rules.

To check the results, we also applied the Holm's step-up and step-down procedure sequentially to test the hypotheses ordered by their significance. The last one is more powerful than the previous Bonferroni Dunn single-step and it makes no additional assumptions about the hypotheses tested ${ }^{47}$. As can be seen in Table 7, accuracy via Holm's test with $\alpha=0.05$ detect the same significant differences between MCGEP and SLAVE, HIDER, Tan, Falco, Bojarczuk and CORE as Bonferroni-Dunn. Holm's at $\alpha=0.05$ does not detect significant differences among MCGEP and GASSIST, LOGIT-BOOST and UCS.

In general, as the Bonferroni-Dunn test shows, the GASSIST method is the most balanced of all the alternative methods analyzed in this work. It also highlights the need to improve the MCGEP method proposed by this paper in regard to the size of the generated models. In the accuracy metric, MCGEP outperformed GASSIST, although the difference is not significant from the point of view of the statistical tests applied (Holm tests, $\alpha=0.05$ ). Taking both criteria (accuracy and model size) at the same time Tan, HIDER and SLAVE are the worst algorithms -see Figure 4. Falco, Bojarzuck and CORE behave well on the size of the generated models, however, they have a mediocre performance in accuracy. UCS and LOGIT-BOOST perform very well in terms of accuracy; in contrast, their performance is worse when attempting to obtain compact models.

As stated above, in statistical terms, when accuracy alone is taken into account, no significant differences were found between MCGEP, GASSIST, LOGIT-BOOST and UCS, although we can make some judgments about the performance of the algorithms in some application scenarios, for example, with variations in the number of classes and in the number of attributes in data sets. Nevertheless, it does not seem necessary to evaluate the variations in the number of instances of data sets.

The comparison was based on information from the individual ranking presented in Table 5. We divided the algorithms into three new ranks, High, Middle (Mid.) and Low. To do so, we attempted to make an equitable division by leaving the same number of ranks in each division and leaving the remaining elements in the Mid. division. For the number of classes, we make the division whenever possible in such a way that it had the same number of data sets in each division; we obtained the following data sets cases: (2) binary; (3-5) between 3 and 5 
Table 7: Holm / Hochberg Table for $\alpha=0.05$

\begin{tabular}{|c|c|c|c|c|}
\hline$i$ & algorithm & $z=\left(R_{0}-R_{i}\right) / S E$ & $p$ & Holm/Hochberg/Hommel \\
\hline 9 & CORE & 5.8229 & $5.7836 \mathrm{E}-9$ & 0.0056 \\
8 & Bojarczuk & 5.7707 & $7.8955 \mathrm{E}-9$ & 0.0063 \\
7 & Falco & 4.9612 & $7.0054 \mathrm{E}-7$ & 0.0071 \\
6 & Tan & 3.3945 & $6.8750 \mathrm{E}-4$ & 0.0083 \\
5 & HIDER & 2.8462 & 0.0044 & 0.0100 \\
4 & SLAVE & 2.6112 & 0.0090 & 0.0125 \\
\hline 3 & UCS & 2.1412 & 0.0323 & 0.0167 \\
2 & LOGIT-BOOST & 2.0106 & 0.0444 & 0.0250 \\
1 & GASSIST & 0.7311 & 0.4647 & 0.0500 \\
\hline III & MCGEP & Control algorithm & III & III \\
\hline
\end{tabular}

classes and (6-11) between 6 and 11 classes. For the number of attributes, we applied the same process but also trying to maximize the distances between one range and other. Attribute ranges (4-9), (13-21) and (30-60) were derived from these divisions and individual ranking taken from Table 5. Tables 8 and 9 have been created to provide better visualization of the count for classes and attributes respectively.

As shown in Table 8, MCGEP algorithm achieves a high level (first three places in the ranking) in 16 of the 20 data sets. In the binary data sets, it achieves the best performance, since it is ranked among the top three in all of them. In the twenty data sets evaluated, this algorithm has no Low performances. In half of the data sets between 7 and 10 classes, its performance is High, while it also achieves Mid. performance in the other half.

The GASSIST algorithm (second in the overallranking) achieves High performance in almost half of the data sets, however in the binary data sets, its performance is basically Mid. It attains the performance of MCGEP in data sets between 3 and 7 classes.Like the previous algorithm, GASSIST also has no Low performance in data sets. The performance of the LOGIT-BOOST algorithm is High in almost half of the data sets, however in the binary and in some many-classes (meaning in our work between 7 and 11 classes) data sets, have a Low performance. Finally, UCS excels over the other four algorithms in many-classes data sets. In this manyclasses data sets, its performance was between Mid. and High.
Table 8: Class distribution count in rank achieved by each algorithm. (2) means binary data sets, (3$5)$ means 3 to 5 class data sets. (6-11) means 6 to 11 class data sets. Algorithm rank High (1-3) mean algorithms that reach a high rank 1,2 or 3 , and so on.

\begin{tabular}{lc|rrr}
\hline \multicolumn{2}{c}{ Algorithm rank } & \multicolumn{3}{c}{ MCGEP } \\
\hline Low & $(8-10)$ & 0 & 0 & 0 \\
Mid. $\quad(4-7)$ & 0 & 1 & 3 \\
High & $(1-3)$ & 8 & 5 & 3 \\
\hline \multicolumn{2}{l}{ Number of class $\mapsto$} & $(2)$ & $(3-5)$ & $(6-11)$ \\
\hline
\end{tabular}

\begin{tabular}{lc|rrr}
\hline \multicolumn{3}{c}{ Algorithm rank } & \multicolumn{3}{c}{ GASSIST } \\
\hline Low $\quad(8-10)$ & 0 & 0 & 0 \\
Mid. $\quad(4-7)$ & 5 & 1 & 5 \\
High $\quad(1-3)$ & 3 & 5 & 1 \\
\hline \multicolumn{2}{l}{ Number of class $\mapsto$} & $(2)$ & $(3-5)$ & $(6-11)$ \\
\hline
\end{tabular}

\begin{tabular}{lc|rrr}
\hline \multicolumn{3}{c}{ Algorithm rank } & \multicolumn{3}{c}{ LOGIT-BOOST } \\
\hline Low & $8-10$ & 2 & 0 & 2 \\
Mid. & $4-7$ & 3 & 3 & 1 \\
High & $1-3$ & 3 & 3 & 3 \\
\hline \multicolumn{2}{l}{ Number of class $\mapsto$} & $(2)$ & $(3-5)$ & $(6-11)$ \\
\hline
\end{tabular}

\begin{tabular}{lc|rrr}
\hline \multicolumn{2}{c}{ Algorithm rank } & \multicolumn{3}{c}{ UCS } \\
\hline Low & $8-10$ & 1 & 2 & 0 \\
Mid. & $4-7$ & 4 & 3 & 2 \\
High & $1-3$ & 3 & 1 & 4 \\
\hline \multicolumn{2}{l}{ Number of class $\mapsto$} & $(2)$ & $(3-5)$ & $(6-11)$ \\
\hline
\end{tabular}


When a similar analysis is performed but this time considering the distribution of attributes (see Table 9), we note that MCGEP excels in data sets of many-attributes (read for this work between 30 and 60 attributes). In four of the five data sets, it achieves High performance and in the fifth a Mid. performance.

Table 9: Attribute distribution counts in rank achieved by each algorithm. (4-9) means data sets with 4 to 9 attributes, (13-21) means data sets with 13 to 21 attributes. (30-60) means data sets with 30 to 60 attributes. Algorithm rank High (1-3) means algorithms that reach a high rank 1,2 or 3 , and so on.

\begin{tabular}{|c|c|c|c|c|}
\hline \multicolumn{2}{|c|}{ Algorithm rank } & \multicolumn{3}{|c|}{ MCGEP } \\
\hline Low & $(8-10)$ & 0 & 0 & 0 \\
\hline Mid. & $(4-7)$ & 3 & 0 & 1 \\
\hline High & $(1-3)$ & 6 & 6 & 4 \\
\hline \multicolumn{2}{|c|}{ Number of class $\mapsto$} & $(4-9)$ & $(13-21)$ & $(30-60)$ \\
\hline \multicolumn{2}{|c|}{ Algorithm rank } & \multicolumn{3}{|c|}{ GASSIST } \\
\hline Low & $(8-10)$ & 0 & 0 & 0 \\
\hline Mid. & $(4-7)$ & 6 & 2 & 3 \\
\hline High & $(1-3)$ & 3 & 4 & 2 \\
\hline \multicolumn{2}{|c|}{ Number of class $\mapsto$} & $(4-9)$ & $(13-21)$ & $(30-60)$ \\
\hline \multicolumn{2}{|c|}{ Algorithm rank } & \multicolumn{3}{|c|}{ LOGIT-BOOST } \\
\hline Low & $8-10$ & 1 & 1 & 2 \\
\hline Mid. & $4-7$ & 1 & 4 & 2 \\
\hline High & $1-3$ & 7 & 1 & 1 \\
\hline \multicolumn{2}{|c|}{ Number of class $\mapsto$} & $\begin{array}{l}(4-9) \\
\end{array}$ & $(13-21)$ & $(30-60)$ \\
\hline \multicolumn{2}{|c|}{ Algorithm rank } & \multicolumn{3}{|c|}{ UCS } \\
\hline Low & $8-10$ & 1 & 2 & 0 \\
\hline Mid. & $4-7$ & 3 & 3 & 3 \\
\hline High & $1-3$ & 5 & 1 & 2 \\
\hline \multicolumn{2}{|c|}{ Number of class $\mapsto$} & $\begin{array}{l}(4-9) \\
\end{array}$ & $(13-21)$ & $(30-60)$ \\
\hline
\end{tabular}

In contrast, the LOGIT-BOOST algorithm has the worst performance in data sets with manyattributes. In the case of MCGEP, its performance is High for attributes between 13 and 21, and often it has High performance with few-attributes (4 to 9). The LOGIT-BOOST algorithm only slightly excels in the case of few-attributes, where most of the time it is the algorithm with High performance.

In summary, the MCGEP algorithm can be said to have good performance in data sets with a variable number of attributes. In consonance with the adjustments made to head size parameter, this indicates that we used a correct heuristic to adjust this parameter $\left(\right.$ head $_{\text {size }} \approx 10 *$ attributes $\left._{\text {number }}\right)$. Nevertheless, we noted a slight reduction in the performance of this algorithm with many-classes data sets, this may be due mainly to the one-vs-all (OVA) approach used. It is well-known that imbalanced training data sets are produced when instances from a single class are compared to all other instances ${ }^{48}$. MCGEP is not completely ready to deal with this undesirable effect. For that reason, two future lines of work have been identified. The first, an implementation of the one-vs-one (OVO) approach and the second, to provide in-built support for this imbalance issue.

XCS and GASSIST were stated in the review ${ }^{17}$ as being "the two most outstanding learners in the GBML history". On the other hand, Orriols-Puig et al. ${ }^{18}$ presented UCS (the evolution of XCS) as the learning algorithm that resulted in the most accurate models on average. Also Orriols-Puig et al. ${ }^{18}$ conclude that GASSIST yielded competitive results in terms of accuracy. We found GASSIST, LOGITBOOST and UCS to provide very similar results in their behavior during our assessment of MCGEP. Therefore we feel it is not fitting to make pairwise comparisons when we only tested whether a newly proposed method is better than existing ones ${ }^{47}$.

\section{Conclusions and further work}

In this paper, we tested and statistically validated the competitiveness of an algorithm for discovering classification rules with gene expression programming (MCGEP) in particular in terms of the accuracy metric. It was built by taking elements from several other inspiring works described in current literature. The product of sensibility, specificity and simplicity was taken from ${ }^{27}$ as a function of fitness, while Token Competition and the 
vision of creating vectors with candidate solutions came from ${ }^{19}$. The vector of candidate solutions was used to drive the evolutionary process by recalling the best rules from the previous generation to the next generation, like the human immunology system remembers previous pathological conditions. The gene expression programming paradigm proposed by Ferreira in ${ }^{10}$ with the adjustments described in detail in ${ }^{21}$ was used in the coding of individuals, which ensures intrinsic efficiency in the evolutionary process. Twenty data sets (numerics or binarized-nominals) were adopted from KEEL and UCI projects. To validate the competitiveness of MCGEP, a comparison was made against nine well known algorithms. The experimental results achieved on twenty benchmark real-world data sets detailed in section 6 show that our approach is significantly better with respect to the accuracy metric than some state-of-the-art genetic rule-based algorithms (i.e., SLAVE, HIDER, Tan, Falco, Bojarczuk and CORE) and not significantly worse than other better algorithms (i.e., GASSIST, LOGIT-BOOST and UCS). Thus, the competitiveness of the GEP approach for discovering classification rules was empirically demonstrated. On the other hand, GASSIST was the most balanced method of all the others assessed in this work. Throughout this study, we have identified the strengths but also the weaknesses of the approach used and thus a great deal of future work remains to be carried out: the possibility of using nominal attributes; the inclusion of the principle of minimum description length (MDL) as used by Zhou et $\mathrm{al}^{20}$ to reduce the slight overfitting that exists in some cases; the treatment of unbalanced data or the implementation of OVO variant to replace the current OVA variant; the possibility of imposing different costs on misclassification. A method for adjusting the parameters $w_{1}$ and $w_{2}$ needs to be researched, as does the implementation of multi-objective version, in order to perform searches of candidate solutions over the Pareto front. Furthermore, we need to make improvements in reducing the number of models generated because it was clearly seen to be one of the weak points of this work.

\section{Acknowledgments}

"This project was funded by the Deanship of Scientific Research (DSR), King Abdulaziz University, Jeddah, under grant No. (2-611-35/HiCi) and the Spanish Ministry of Economy and Competitiveness and FEDER funds under grant No. TIN2014-55252P. The authors therefore express their sincerest gratitude for such technical and financial support".

\section{References}

1. John Ross Quinlan. C4.5: Programs for Machine Learning. Morgan Kaufmann Publishers Inc., San Francisco, CA, USA, 1993.

2. Vladimir Naumovich Vapnik. The Nature of Statistical Learning Theory. Springer-Verlag New York, Inc., New York, NY, USA, 1995.

3. George John and Pat Langley. Estimating Continuous Distributions in Bayesian Classifiers. In In Proceedings of the Eleventh Conference on Uncertainty in Artificial Intelligence, pages 338-345. Morgan Kaufmann, 1995.

4. David Edward Goldberg. Genetic Algorithms in Search, Optimization and Machine Learning. Addison-Wesley Longman Publishing Co., Inc., Boston, MA, USA, 1st edition, 1989.

5. John Reed Koza. Genetic Programming: On the Programming of Computers by Means of Natural Selection. MIT Press, Cambridge, MA, USA, 1992.

6. Agoston Endre Eiben and James Edward Keith Smith. Introduction to Evolutionary Computing. SpringerVerlag, 2003.

7. John Henry Holland. Adaptation in Natural and Artificial Systems: An Introductory Analysis with Applications to Biology, Control and Artificial Intelligence. MIT Press, Cambridge, MA, USA, 1992.

8. Zbigniew Michalewicz. Genetic Algorithms + Data Structures $=$ Evolution Programs. Springer-Verlag New York, Inc., New York, NY, USA, 2nd edition, 1994.

9. Pedro G. Espejo, Sebastián Ventura, and Francisco Herrera. A Survey on the Application of Genetic Programming to Classifcation. Ieee Transactions on Systems, Man, and Cybernetics, Part C: Applications and Reviews, 40(2):121-144, 2010.

10. Cândida Ferreira. Gene Expression Programming: a New Adaptive Algorithm for Solving Problems. Complex Systems, 13(2):87-129, 2001.

11. John Henry Holland and Judith Olson Reitman. Cognitive Systems Based on Adaptive Algorithms. Intelligence SIGART Bulletin, 63:49-49, June 1977. 
12. Kenneth Alan De Jong. Learning Concept Classification Rules using Genetic Algorithms. In Proceedings of the Twelfth International Joint Conference on Artificial Intelligence, pages 651-656. Morgan Kaufmann, 1991.

13. Stephen Frederick Smith. A Learning System Based on Genetic Adaptive Algorithms. PhD thesis, Pittsburgh, PA, USA, 1980. AAI8112638.

14. Gilles Venturini. SIA: A Supervised Inductive Algorithm with Genetic Search for Learning Attributes based Concepts. In Pavel Brazdil, editor, ECML, volume 667 of Lecture Notes in Computer Science, pages 280-296. Springer, 1993.

15. Attilio Giordana and Filippo Neri. Search-intensive Concept Induction. Evol. Comput., 3(4):375-416, December 1995.

16. David Perry Greene and Stephen Frederick Smith. Competition-Based Induction of Decision Models from Examples. Mach. Learn., 13(2-3):229-257, November 1993.

17. Alberto Fernández, Salvador García, Julián Luengo, Ester Bernadó-Mansilla, and Francisco Herrera. Genetics-based machine learning for rule induction: State of the art, taxonomy, and comparative study. IEEE Transactions on Evolutionary Computation, 14(6):913-941, 2010.

18. Albert Orriols-Puig, Jorge Casillas, and Ester Bernadó-Mansilla. Genetic-based machine learning systems are competitive for pattern recognition. Evol. Intel., pages 1-24, 2008.

19. Kay Chen Tan, Arthur Tay, Tong Heng Lee, and C. M. Heng. Mining multiple comprehensible classification rules using genetic programming. In David B. Fogel, Mohamed A. El-Sharkawi, Xin Yao, Garry Greenwood, Hitoshi Iba, Paul Marrow, and Mark Shackleton, editors, Proceedings of the 2002 Congress on Evolutionary Computation CEC2002, pages 1302 1307. IEEE Press, 2002.

20. Chi Zhou, Weimin Xiao, Thomas Michael Tirpak, and Peter C. Nelson. Evolving Accurate and Compact Classification Rules with Gene Expression Programming. Trans. Evol. Comp, 7(6):519-531, 2003.

21. Cândida Ferreira. Gene Expression Programming: Mathematical Modeling by an Artificial Intelligence. Revised and extended edition. Springer, 2nd edition, May 2006.

22. Durga Prasad Muni, Nikhil Ranjan Pal, and Jyotirmoy Das. A Novel Approach to Design Classifiers Using Genetic Programming. Trans. Evol. Comp, 8(2):183196, April 2004.

23. Andrew Webb. Statistical Pattern Recognition. John Wiley \& Sons, 2nd edition, 2002.

24. Ronald Linn Rivest. Learning Decision Lists. Machine Learning, 2(3):229-246, 1987.

25. Wolfgang Banzhaf, Frank Francone, Robert Keller, and Peter Nordin. Genetic Programming: An Introduction: on the Automatic Evolution of Computer Programs and Its Applications. Morgan Kaufmann Publishers Inc., San Francisco, CA, USA, 1998.

26. Man Leung Wong and Kwong Sak Leung. Data Mining Using Grammar-Based Genetic Programming and Applications. Kluwer Academic Publishers, Norwell, MA, USA, 2000.

27. Celia Cristina Bojarczuk, Heitor Silvério Lopes, Alex Alves Freitas, and Edson Luiz Michalkiewicz. A Constrained-syntax Genetic Programming System for Discovering Classification Rules: Application to Medical Data Sets. Artif. Intell. Med., 30(1):27-48, January 2004.

28. David Hand. Construction and Assessment of Classification Rules. Wiley, 3rd edition, 1997.

29. Heitor Silvério Lopes, Mario S. Coutinho, and Walter C. Lima. An evolutionary approach to simulate cognitive feedback learning in medical domain. In E. Sanchez, T. Shibata, and L. A. Zadeh, editors, Genetic Algorithms and Fuzzy Logic Systems, volume 7 of Advances in Fuzzy Systems - Applications and Theory, pages 193-207. World Scientific Publishing, 1997.

30. Jaume Bacardit. Pittsburgh genetic-based machine learning in the data mining era: representations and generalization and run-time. $\mathrm{PhD}$ thesis, Dept. of Department of Computer Science, University Ramon LLull, December 2004.

31. Ester Bernadó-Mansilla and Josep Maria GarrellGuiu. Accuracy-based Learning Classifier Systems: Models, Analysis and Applications to Classification Tasks. Evol. Comput., 11(3):209-238, September 2003.

32. Stewart Wilson. Get Real! XCS with ContinuousValued Inputs. In Learning Classifier Systems, From Foundations to Applications, pages 209-222, London, UK, UK, 2000. Springer-Verlag.

33. Kenneth Alan De Jong, William M. Spears, and Diana Faye Gordon. Using genetic algorithms for concept learning. Machine Learning, 13(2-3):161-188, Nov.Dec. 1993.

34. Jesús Salvador Aguilar-Ruiz, José C. Riquelme, and Miguel Toro. Evolutionary Learning of Hierarchical Decision Rules. Trans. Sys. Man Cyber. Part B, 33(2):324-331, April 2003.

35. Jesús Salvador Aguilar-Ruiz, Raúl Giráldez, and José C. Riquelme. Natural encoding for evolutionary supervised learning. IEEE Transactions on Evolutionary Computation, 11(4):466-479, August 2007.

36. Antonio González and Raúl Pérez. SLAVE: A Genetic Learning System Based on an Iterative Approach. Trans. Fuz Sys., 7(2):176-191, April 1999.

37. Jos Otero and Luciano Snchez. Induction of descriptive fuzzy classifiers with the logitboost algorithm. 
Soft Computing, 10(9):825-835, 2006.

38. Yoav Freund and Robert Elias Schapire. Experiments with a New Boosting Algorithm. In Proceedings of the Thirteenth International Conference, pages 148-156, 1996.

39. Jerome H. Friedman, Trevor J. Hastie, and Robert Tibshirani. Additive logistic regression: a statistical view of boosting. Ann Stat, 32(2):337-374, 2000.

40. K. C. Tan, Q. Yu, and J. H. Ang. A coevolutionary algorithm for rules discovery in data mining. International Journal of Systems Science, 37(12):835-864, 2006.

41. Ivanoe De Falco, Antonio Della Cioppa, and Ernesto Tarantino. Discovering interesting classification rules with genetic programming. Applied Soft Computing, 1:257-269, 2002.

42. Jesús Alcalá-Fdez, Alberto Fernández, Julián Luengo, Joaquin Derrac, Salvador García, Luciano Sánchez, and Francisco Herrera. KEEL Data-Mining Software Tool: Data Set Repository and Integration of Algorithms and Experimental Analysis Framework. Journal of Multiple-Valued Logic and Soft Computing, 17(2-3):255-287, 2011.

43. M. Lichman. UCI Machine Learning Repository, 2013.
44. Sebastián Ventura, Cristóbal Romero, Amelia Zafra, José; A. Delgado, and César Hervás. JCLEC: A Java Framework for Evolutionary Computation. Soft Computing, 12(4):381-392, October 2007.

45. Wagner Rodrigo Weinert and Heitor Silvério Lopes. GEPCLASS: A classification Rule Discovery Tool Using Gene Expression Programming. Lecture Notes in Artificial Intelligence, (4093):871-880, 2006.

46. Jesús Alcalá-Fdez, Luciano Sánchez, Salvador García, María José del Jesús, Sebastian Ventura, Josep Maria Garrell, José Otero, Cristóbal Romero, Jaume Bacardit, Victor M. Rivas, Juan Carlos Fernández, and Francisco Herrera. KEEL : a software tool to assess evolutionary algorithms for data mining problems. Soft Computing, 13(3):307-318, 2008.

47. Janez Demšar. Statistical Comparisons of Classifiers over Multiple Data Sets. Machine Learning Research, 7:1-30, 2006.

48. Mikel Galar, Alberto Fernández, Edurne Barrenechea, Humberto Bustince, and Francisco Herrera. An overview of ensemble methods for binary classifiers in multi-class problems: Experimental study on onevs-one and one-vs-all schemes. Pattern Recognition, 44(8):1761-1776, 2011. 